Article

\title{
A Novel AlGaN/GaN Transient Voltage Suppression Diode with Bidirectional Clamp Capability
}

\author{
Zhiyuan He, Yijun Shi *D, Yun Huang, Yiqiang Chen*D, Hongyue Wang, Lei Wang, Guoguang Lu and Yajie Xin
}

Citation: He, Z.; Shi, Y.; Huang, Y.; Chen, Y.; Wang, H.; Wang, L.; Lu, G.; Xin, Y. A Novel AlGaN/GaN Transient Voltage Suppression Diode with Bidirectional Clamp Capability. Micromachines 2022, 13, 299. https:// doi.org/10.3390/mi13020299

Academic Editors: Alessandro Chini and Nicolò Zagni

Received: 13 January 2022

Accepted: 11 February 2022

Published: 14 February 2022

Publisher's Note: MDPI stays neutral with regard to jurisdictional claims in published maps and institutional affiliations.

Copyright: (C) 2022 by the authors. Licensee MDPI, Basel, Switzerland. This article is an open access article distributed under the terms and conditions of the Creative Commons Attribution (CC BY) license (https:// creativecommons.org/licenses/by/ $4.0 /)$.
The Science and Technology on Reliability Physics and Application of Electronic Component Laboratory, China Electronic, Product Reliability and Environmental Testing Research Institute, Guangzhou 510610, China; hezhiyuan1988@126.com (Z.H.); huangyun@ceprei.com (Y.H.); wanghongyue@pku.edu.cn (H.W.); leiwang@ceprei.com (L.W.); luguog@126.com (G.L.); 201811022423@std.uestc.edu.cn (Y.X.)

* Correspondence: syj20094870@sina.com (Y.S.); yiqiang-chen@hotmail.com (Y.C.); Tel.: +86-185-837-12009 (Y.S.)

\begin{abstract}
This work proposes a novel AlGaN/GaN transient voltage suppression (TVS) diode (BTVS-D) with bidirectional clamp capability, which consists of a small-size AlGaN/GaN monolithic bidirectional switch, two 2DEG-based current-limiting resistors $\left(R_{1 \mathrm{~A}} / R_{1 \mathrm{C}}\right.$, in parallel connection between the gate electrodes and the neighboring ohmic-contact electrodes (anode/cathode)), and a 2DEG-based proportional amplification resistor $\left(R_{2}\right.$, in parallel connection between two gate electrodes). It is demonstrated that the proposed B-TVS-D possesses a symmetrical triggering voltage $\left(V_{\text {trig }}\right)$ and a high secondary breakdown current $\left(I_{\mathrm{S}}\right.$, over $8 \mathrm{~A}$, corresponding to $12 \mathrm{kV}$ human body model failure voltage) in different directional electrostatic discharge (ESD) events. The proposed diode can effectively enhance the electrostatic discharge robustness for the GaN-based power system. It is also verified that $R_{1 \mathrm{~A}} / R_{1 \mathrm{C}}$ and $R_{2}$ have an important impact on $V_{\text {trig }}$ of the proposed B-TVS-D. Both the decrease in $R_{2}$ and increase in $R_{1 \mathrm{~A}} / R_{1 \mathrm{C}}$ can lead to the decrease of $V_{\text {trig. }}$. In addition, the proposed B-TVS-D can be fabricated on the conventional p-GaN HEMT platform, making the ESD design of the GaN-based power system more convenient.
\end{abstract}

Keywords: transient voltage suppression; TVS diode; GaN HEMT; Transmission Line Pulsing

\section{Introduction}

Gallium nitride $(\mathrm{GaN})$ has several notable material properties (such as high electron saturation velocity, high electron mobility, and wide bandgap), which makes GaN-based diodes and high electron mobility transistors (HEMTs) attract broad attention in power electronics [1-6]. However, the possibility of an electrostatic discharge (ESD) event always threatens the reliability of GaN-based HEMTs and diodes [7-15]. In our previous study, it is found that the human body model failure voltage $\left(V_{\mathrm{HBM}}\right)$ of the p-gate structure of some commercial HEMTs is less than $0.5 \mathrm{kV}$, which is far below the trade standard $(2 \mathrm{kV})[16,17]$. Some researchers have studied and analyzed the ESD protection ability of a GaN-based Schottky barrier diode [11-15]. Although GaN-based Schottky barrier diodes can discharge the positive electrostatic charge, the forward triggering voltage of GaN-based Schottky barrier diodes is less than $2 \mathrm{~V}$, which is lower than the working voltage $(+6 \mathrm{~V})$ of p-GaN HEMT. In addition, GaN-based Schottky barrier diodes can discharge forward electrostatic charge but cannot discharge reverse electrostatic charge. Thus, GaN-based Schottky barrier diodes cannot effectively protect the gate structure of commercial p-GaN HEMT from ESD damage. To effectively protect the $\mathrm{p}-\mathrm{GaN}$ HEMTs from ESD damage, it is necessary to ameliorate the ESD robustness for the p-gate structure. In this connection, Yajie Xin has proposed a unidirectional AlGaN/GaN transient voltage suppression (TVS) diode [18], which can be self-triggered by a desirable value in the unidirectional transient ESD event. However, in some fields, namely AC circuit and communication systems, a TVS diode with the capability of bidirectional transient voltage suppression is needed [19]. To achieve this goal, we have proposed a bidirectional AlGaN/GaN TVS diode, which possesses a 
symmetrical triggering voltage $\left(V_{\text {trig }}\right)$ and a high secondary breakdown current $\left(I_{\mathrm{s}}\right)$ in different directional electrostatic discharge events [19]. However, to obtain a required triggering voltage, a relatively large capacitor is needed, which will also obviously decrease the $I_{\mathrm{S}}$ of the bidirectional AlGaN/GaN TVS diode; i.e., the protection capability of that bidirectional AlGaN/GaN TVS diode will be obviously weakened, as described in our previous work. Therefore, there still is an urgent need for optimizing or redesigning the bidirectional AlGaN/GaN TVS diode.

In this work, we have proposed a novel bidirectional AlGaN/GaN TVS diode (BTVS-D), which consists of a small-size AlGaN/GaN monolithic bidirectional switch (MBS), two current-limiting resistors $\left(R_{1 \mathrm{~A}} / R_{1 \mathrm{C}}\right.$, in parallel connection between the gate electrodes and the neighboring ohmic-contact electrodes (anode/cathode)), and a 2DEG-based proportional amplification resistor $\left(R_{2}\right.$, in parallel connection between two gate electrodes). The proposed B-TVS-D possesses a symmetrical $V_{\text {trig }}$ and a high $I_{\mathrm{s}}$ in different directional electrostatic discharge events. This work is organized as follows. First, the structures and mechanisms of the unidirectional TVS diode and the proposed B-TVS-D are presented. Then, the characteristics of the proposed B-TVS-D, and the influence of $R_{1 \mathrm{C}} / R_{1 \mathrm{~A}}$ and $R_{2}$ are investigated. Finally, the conclusions are drawn.

\section{Materials and Methods}

Before introducing the proposed AlGaN/GaN B-TVS-D, it is necessary to introduce the unidirectional AlGaN/GaN TVS diode developed in previous work [18]. Figure 1a, b give the schematic structure of the unidirectional TVS diode, containing a p-GaN HEMT, a 2DEGbased current-limiting resistor $\left(R_{1}\right)$ and a 2DEG-based proportional amplification resistor $\left(R_{2}\right) . R_{1}$ is parallelly connected between the diode's gate electrode and the cathode electrode, and $R_{2}$ is parallelly connected between the diode's gate electrode and the anode electrode. It is obvious that the unidirectional TVS diode can be fabricated on the conventional p-GaN HEMT platform.

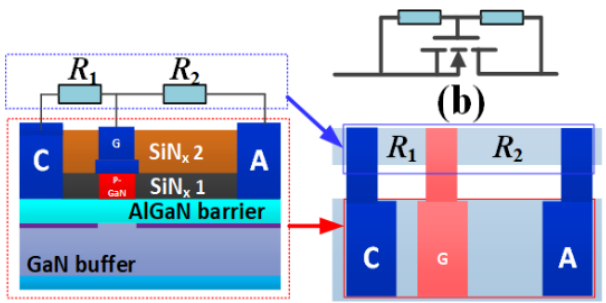

(a)

(c)

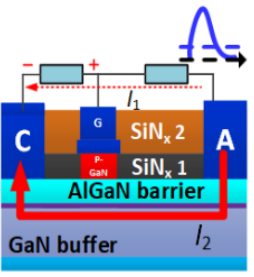

(d)

Figure 1. (a) The structure, (b) equivalent circuit, (c) plane layout and (d) working mechanism of the unidirectional TVS diode [18].

The mechanism of the unidirectional TVS diode is exhibited in Figure 1d. During the forward transient ESD event (from A to C), a small amount transient electrostatic charges will flow through $R_{1}$ and $R_{2}$. Then, there is a transient voltage drop between the diode's gate and the cathode. When the transient voltage drop is larger than the threshold voltage $\left(V_{\text {th }}\right)$ of the p-GaN HEMT, the unidirectional TVS diode will be triggered. Then, the forward transient electrostatic charges can be discharged through the unidirectional TVS diode (Figure $1 \mathrm{~d}$ ). Thereby, the ESD damage can be avoided. As can be surmised, $R_{1}$ and $R_{2}$ play an important role on the forward triggering voltages $\left(V_{\text {trig_F}}\right)$ of the unidirectional TVS diode. The increase in $R_{1}$ or decrease in $R_{2}$ can lead to the decrease in $V_{\text {trig_F }}$ (Figure 2) [18]. So, through changing $R_{1}$ or $R_{2}$, a desirable $V_{\text {trig_F }}$ can also be obtained. However, in the reverse ESD event (from $C$ to $A$ ), the unidirectional TVS diode is triggered by a very low voltage, just like a lateral field effect rectifier (L-FER). And the changes in $R_{1}$ and $R_{2}$ have nearly no effect on the device's reverse triggering voltages $\left(V_{\text {trig_R }}\right)$. So, the unidirectional TVS diode is incapable of clamping the potential to be a desirable voltage in the reverse ESD event. To realize the target of bidirectional clamp, a bidirectional AlGaN/GaN TVS diode is proposed in this work. 

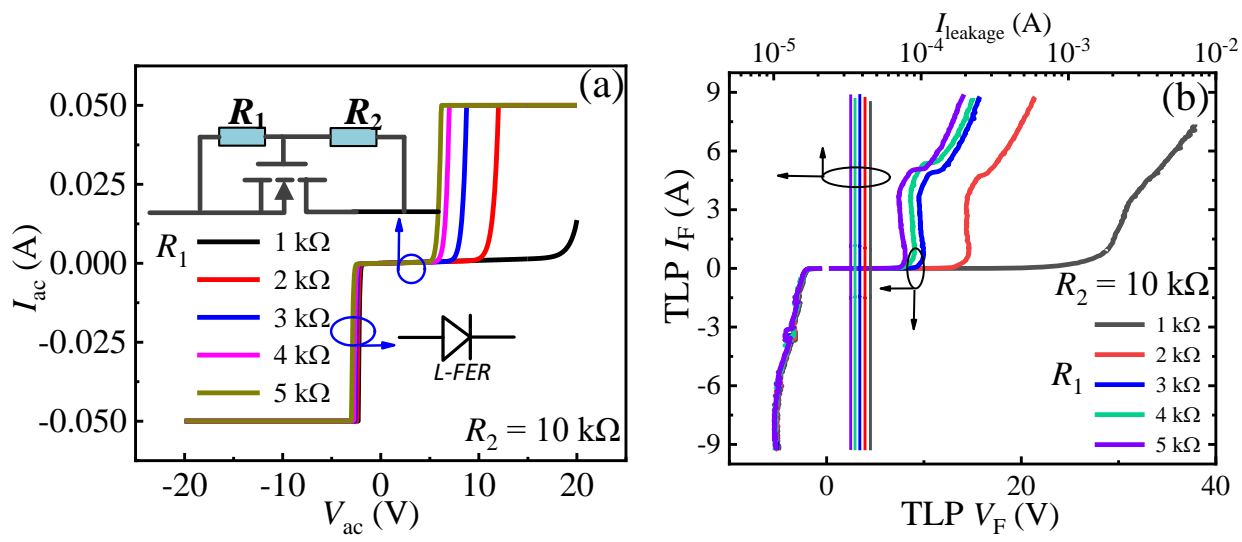

Figure 2. The bidirectional static leakage (a) and TLP I-V characteristics (b) of the unidirectional TVS diode with $R_{2}=10 \mathrm{k} \Omega$ and different $R_{1}$.

Figure $3 \mathrm{a}, \mathrm{b}$ give the structure of the proposed B-TVS-D. The diode consists of a small-size AlGaN/GaN MBS, two 2DEG-based current-limiting resistors $\left(R_{1 \mathrm{C}} / R_{1 \mathrm{~A}}\right)$, and a $2 \mathrm{DEG}$-based proportional amplification resistor $\left(R_{2}\right) . R_{1 \mathrm{C}} / R_{1 \mathrm{~A}}$ is in parallel connection between the gate electrodes and the neighboring ohmic-contact electrodes (anode/cathode), and $R_{2}$ is connected in parallel between two gate electrodes. It can also be found that the proposed B-TVS-D can be fabricated on the traditional p-GaN HEMT platform. Moreover, the required 2DEG-based resistors can be easily integrated through changing the length of the 2DEG-based resistors. For example, when the width of the 2DEG-based resistor is $3 \mu \mathrm{m}$, the required length of the 2DEG-based resistor is less than $100 \mu \mathrm{m}$, and the corresponding area is less than $0.0003 \mathrm{~mm}^{2}$, which makes up no more than $0.01 \%$ of the conventional HEMT's area [20].

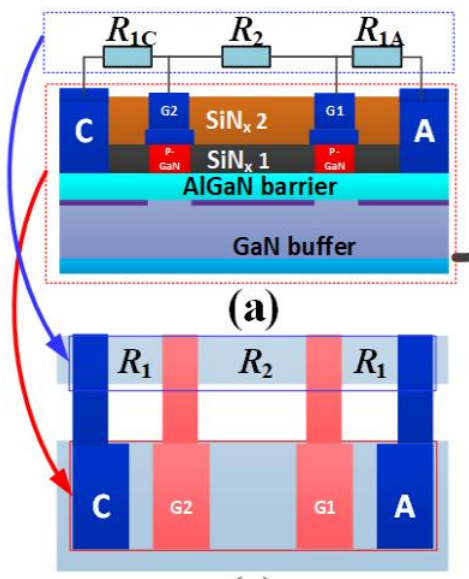

(c)

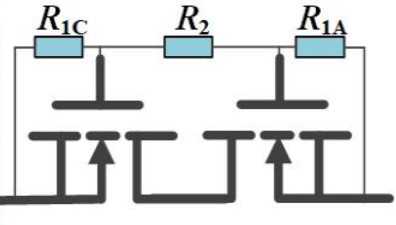

(b)

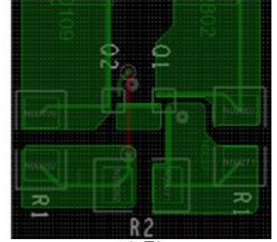

(d)

Figure 3. (a) The structure, (b) equivalent circuit and (c) plane layout of the proposed B-TVS-D. (d) The equivalent structure configured by the chip resistor and the p-GaN HEMT.

The mechanism of the proposed B-TVS-D is exhibited in Figure 4. In both the forward and reverse ESD event, the proposed B-TVS-D is similar to the combination of a unidirectional TVS diode and a L-FER, but in different directions. During the forward ESD event, the diode's first gate structure and anode act as the L-FER, and the diode's second gate structure, cathode, $R_{1 \mathrm{C}}, R_{1 \mathrm{~A}}$ and $R_{2}$ act as the unidirectional AlGaN/GaN TVS diode (Figure $4 a$ ). The L-FER can be turned on at a very low voltage, as shown in Figure 2. The transient electrostatic charges will arouse a forward current flowing through $R_{1 \mathrm{C}}, R_{1 \mathrm{~A}}$ and $R_{2}$, and lead to a transient voltage drop between the diode's second gate electrode and cathode electrode. When the transient voltage drop is larger than $V_{\text {th }}$ of the second gate 
structure of the AlGaN/GaN MBS, the MBS will be turned on. Then, the forward transient electrostatic charge can be released through the proposed B-TVS-D. Through changing $R_{1 C}$, $R_{1 \mathrm{~A}}$ and $R_{2}$, the diode can be triggered by a desirable value in the forward ESD event, just as for the unidirectional TVS diode. Similarly, the proposed B-TVS-D can also be triggered by a desirable value in the reverse ESD event. During the reverse ESD event, the diode's second gate structure and cathode act as the L-FER, and the diode's first gate structure, anode, $R_{1 \mathrm{C}}, R_{1 \mathrm{~A}}$ and $R_{2}$ act as the unidirectional TVS diode. The transient voltage induced by the electrostatic charges will arouse a reverse current flowing through $R_{1 \mathrm{C}}, R_{1 \mathrm{~A}}$ and $R_{2}$, and lead to a transient voltage drop between the diode's first gate electrode and the anode electrode. When the voltage drop is larger than $V_{\text {th }}$ of the first gate structure of the $\mathrm{AlGaN} / \mathrm{GaN}$ MBS, it will be turned on. Then, the reverse transient electrostatic charge can discharge through the proposed B-TVS-D.

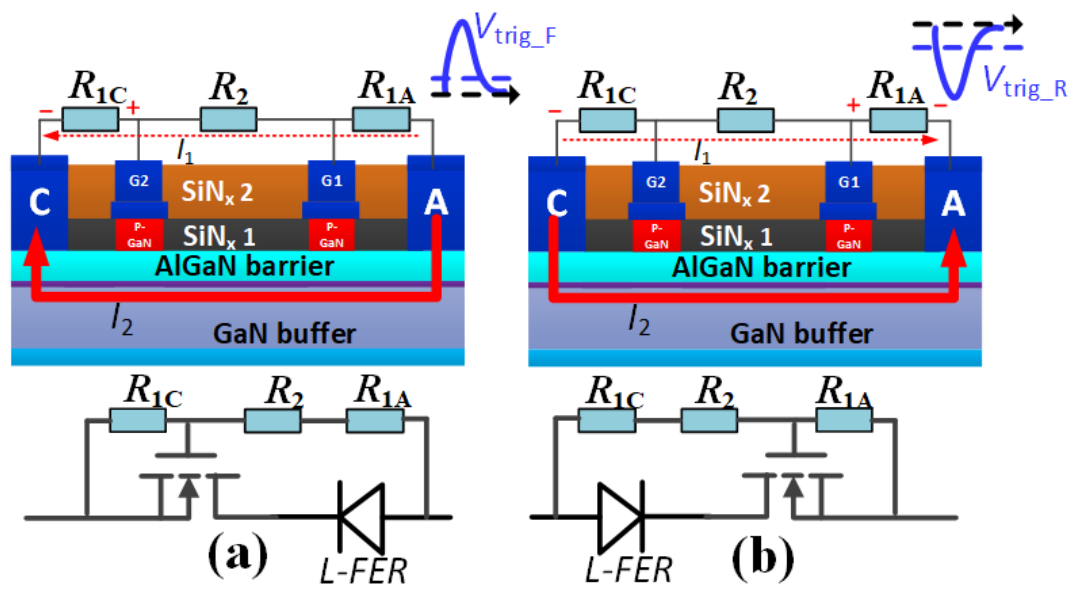

Figure 4. Working mechanism of the proposed AlGaN/GaN B-TVS-D: (a) During the forward ESD event, (b) During the reverse ESD event.

To reduce the validation cost, the working mechanism of the proposed B-TVS-D is verified by the equivalent structure configured by the commercial p-GaN HEMT (EPC2036) [21] and the chip resistor, as shown in Figure 3d. The areas of EPC2036 and the 2DEG-based resistors are only about $0.81 \mathrm{~mm}^{2}$ and $0.0003 \mathrm{~mm}^{2}$, respectively. So, the area of the proposed B-TVS-D is about $1.6203 \mathrm{~mm}^{2}$, which makes up no more than $5 \%$ of the conventional HEMT's area [20]. In this work, the proposed B-TVS-D is tested by our self-developed transmission line pulsing (TLP) measurement system. The rising time and pulse width are set to be $2 \mathrm{~ns}$ and $100 \mathrm{~ns}$, respectively. Since the practical $I_{\mathrm{S}}$ of the proposed B-TVS-D cannot be obtained for the limitation of our self-developed TLP measurement system, $I_{\mathrm{S}}$ in this work is defined at the transient applied voltage reaching to system's limit (1000 V). $V_{\text {trig }}$ of the proposed B-TVS-D is defined at the transient TLP current of $0.1 \mathrm{~A}$.

\section{Results and Discussion}

Figure 5 plots the bidirectional static leakage and TLP I- $V$ characteristic for the proposed B-TVS-D with $R_{1 \mathrm{C}} / R_{1 \mathrm{~A}}=4 \mathrm{k} \Omega$ and $R_{2}=10 \mathrm{k} \Omega$. As stated above, in both the forward and reverse transient ESD event, the proposed B-TVS-D is similar to the combination of the unidirectional TVS diode and L-FER, but in different directions. Thereby, the proposed diode can possess a symmetrical bidirectional static leakage current characteristic and a symmetrical bidirectional TLP I- $V$ characteristic, which are different from those of the unidirectional TVS diode. For the proposed B-TVS-D with $R_{1 \mathrm{C}} / R_{1 \mathrm{~A}}=4 \mathrm{k} \Omega$ and $R_{2}=10 \mathrm{k} \Omega$, the diode's forward turn-on voltage of the static leakage current $\left(V_{T_{-}}\right.$, defined at the static leakage current of $1 \mathrm{~mA})$ is $7.9 \mathrm{~V}$, which is close to its reverse turn-on voltage $\left(V_{\mathrm{T} \_\mathrm{R}}=-7.4 \mathrm{~V}\right.$, defined at the static leakage current of $-1 \mathrm{~mA}$ ). For the unidirectional AlGaN/GaN TVS diode with $R_{1}=4 \mathrm{k} \Omega$ and $R_{2}=10 \mathrm{k} \Omega$, the diode's $V_{\mathrm{T}} \mathrm{F}$ is about $5 \mathrm{~V}$, and the value is different from its $V_{\mathrm{T} \_\mathrm{R}}(\sim-2 \mathrm{~V})$. Thereby, the proposed AlGaN/GaN B-TVS-D with $R_{1 \mathrm{C}} / R_{1 \mathrm{~A}}=4 \mathrm{k} \Omega$ 
and $R_{2}=10 \mathrm{k} \Omega$ will not obviously increase the leakage for GaN-based power system with the static applied voltage in the range from $-7.4 \mathrm{~V}$ to $7.9 \mathrm{~V}$. Through changing $R_{1 \mathrm{C}}, R_{1 \mathrm{~A}}$ and $R_{2}$, a desirable turn-on voltage $\left(V_{\mathrm{T}} \mathrm{F}\right.$ and $V_{\mathrm{T} \_\mathrm{R}}$ ) can be acquired for the proposed B-TVS-D, which will be described in the following part.
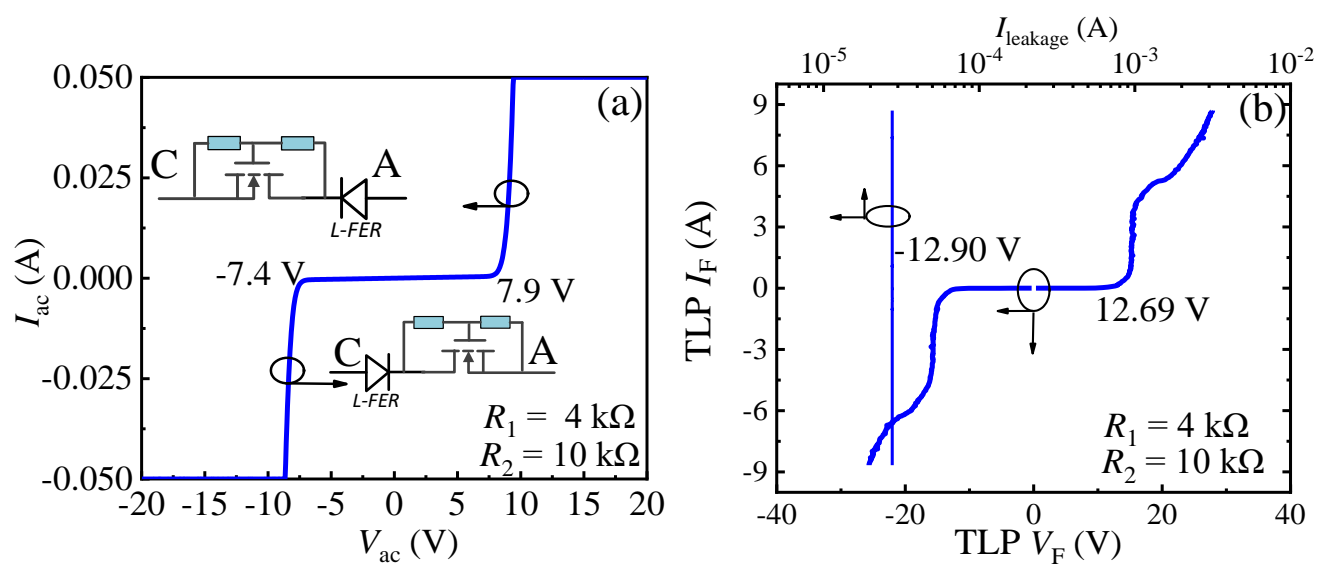

Figure 5. The bidirectional static leakage (a) and TLP I-V characteristic (b) of the proposed B-TVS-D, with $R_{1 \mathrm{C}} / R_{1 \mathrm{~A}}=4 \mathrm{k} \Omega$ and $R_{2}=10 \mathrm{k} \Omega$.

In the positive TLP test, it is found that the proposed B-TVS-D with $R_{1 \mathrm{C}} / R_{1 \mathrm{~A}}=4 \mathrm{k} \Omega$ and $R_{2}=10 \mathrm{k} \Omega$ can be triggered by a low voltage of $12.69 \mathrm{~V}$; the value is close to its $V_{\text {trig_R }}$ $(=-12.9 \mathrm{~V})$. Thus, in different directional transient electrostatic discharge events, the proposed diode can effectively clamp the potential to a low value. Through changing $R_{1 \mathrm{C}}, R_{1 \mathrm{~A}}$ and $R_{2}$, a desirable triggering voltage $\left(V_{\text {trig_F }}\right.$ and $\left.V_{\text {trig } \_}\right)$can be acquired for the proposed B-TVS-D, which is different from the unidirectional TVS diode and will be described in the following part. Besides, in both the different directional TLP tests, the proposed B-TVS-D possesses a high $I_{S}$ of over $8 \mathrm{~A}$, showing that the proposed diode can usefully discharge the transient electrostatic charges in different directional transient electrostatic discharge events. Thus, the proposed diode can effectively enhance the electrostatic discharge robustness for the GaN-based power system.

It can be easily speculated that $R_{1 \mathrm{C}} / R_{1 \mathrm{~A}}$ and $R_{2}$ pay an important role on the bidirectional static leakage and TLP I- $V$ characteristics of the proposed B-TVS-D. Therefore, the characteristics of the proposed B-TVS-D with different $R_{1 \mathrm{C}} / R_{1 \mathrm{~A}}$ and $R_{2}$ are investigated here. First, the bidirectional static leakage and TLP $I-V$ characteristics of the proposed B-TVS-D with different $R_{2}$ are presented in Figures 6 and 7. From Figure 6, it can be seen that the turn-on voltage of the static bidirectional leakage current $\left(V_{\mathrm{T}}\right)$ is increased with the increase in $R_{2}$. With $R_{2}$ increased from $6 \mathrm{k} \Omega$ to $20 \mathrm{k} \Omega, V_{\mathrm{T}}$ are increased from $6.4 \mathrm{~V}$ to $11.2 \mathrm{~V}$ for the proposed AlGaN/GaN B-TVS-D with $R_{1 \mathrm{C}} / R_{1 \mathrm{~A}}=4 \mathrm{k} \Omega$, and increased from $8.2 \mathrm{~V}$ to $18.5 \mathrm{~V}$ for the diode with $R_{1 \mathrm{C}} / R_{1 \mathrm{~A}}=2 \mathrm{k} \Omega$. This is because the increase in $R_{2}$ will decrease the voltage drop at $R_{1 C}$ and $R_{1 \mathrm{~A}}$, subsequently reducing the voltage at the second gate structure in the forward conduction state or reducing the voltage at the first gate structure in the reverse conduction state. Therefore, a higher applied voltage is needed to turn on the normally-off gate structure of the AlGaN/GaN MBS in the proposed B-TVS-D. Hence, through changing $R_{2}$, a desirable $V_{\mathrm{T}}$ can be acquired for the proposed B-TVS-D.

Similarly, in the transient ESD event, the forward and reverse triggering voltages ( $V_{\text {trig_F }}$ and $V_{\text {trig_R }}$ ) of the proposed B-TVS-D are also increased with the increase in $R_{2}$. With $R_{2}$ increased from $6 \mathrm{k} \Omega$ to $20 \mathrm{k} \Omega$, the triggering voltages are increased from $9.2 \mathrm{~V}$ to $19.0 \mathrm{~V}$ for the proposed B-TVS-D with $R_{1 \mathrm{C}} / R_{1 \mathrm{~A}}=4 \mathrm{k} \Omega$, and increased from $11 \mathrm{~V}$ to $25.9 \mathrm{~V}$ for the proposed B-TVS-D with $R_{1 \mathrm{C}} / R_{1 \mathrm{~A}}=2 \mathrm{k} \Omega$. So, through changing $R_{2}$, a desirable triggering voltage can also be acquired for the proposed B-TVS-D (Figure 8). It should be noted that the proposed B-TVS-D with low triggering voltage will possess a low turn-on voltage of the static leakage current. The designers should try to avoid premature turn-on of the static leakage current before obtaining low triggering voltage in the transient ESD 
event. All the proposed B-TVS-D possess a high $I_{\mathrm{S}}$ over than $8 \mathrm{~A}$. Correspondingly, the equivalent HBM failure voltage $\left(V_{\mathrm{HBM}}=I_{\mathrm{S}} \times 1500 \Omega\right)$ of the proposed B-TVS-D reaches to $12 \mathrm{kV}$; the value is higher than that of the bidirectional TVS diode in our previous work [19]. For the bidirectional AlGaN/GaN TVS diode in our previous work, both its $V_{\text {trig }}$ and $I_{S}$ are dependent on its capacitor. With its capacitor increasing from $5 \mathrm{pF}$ to $25 \mathrm{pF}$, its $V_{\text {trig }}$ is decreased from $18 \mathrm{~V}$ to $7 \mathrm{~V}$, but its $I_{S}$ is also decreased from $7 \mathrm{~A}$ to $3 \mathrm{~A}$. Thus, to obtain a required triggering voltage, the diode's protection capability will be weakened. To increase $I_{\mathrm{S}}$ for that bidirectional AlGaN/GaN TVS diode, a lager chip size is needed, which will increase corresponding costs. However, the relatively low static leakage current of the bidirectional AlGaN/GaN TVS diode may attract interest in some application.
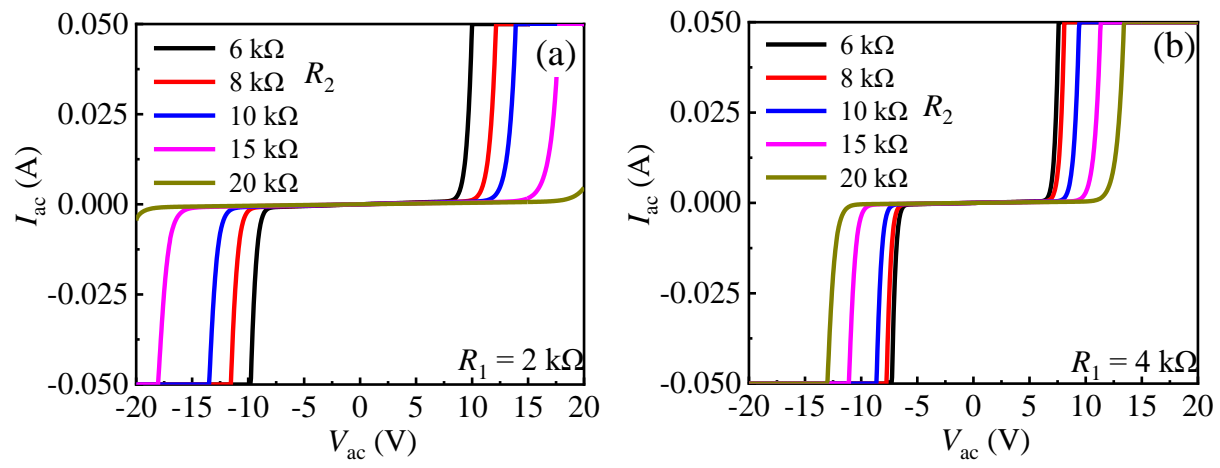

Figure 6. The bidirectional static leakage of the proposed B-TVS-D with different $R_{2}$ : (a) $R_{1 \mathrm{C}} / R_{1 \mathrm{~A}}=2 \mathrm{k} \Omega$; (b) $R_{1 \mathrm{C}} / R_{1 \mathrm{~A}}=4 \mathrm{k} \Omega$.
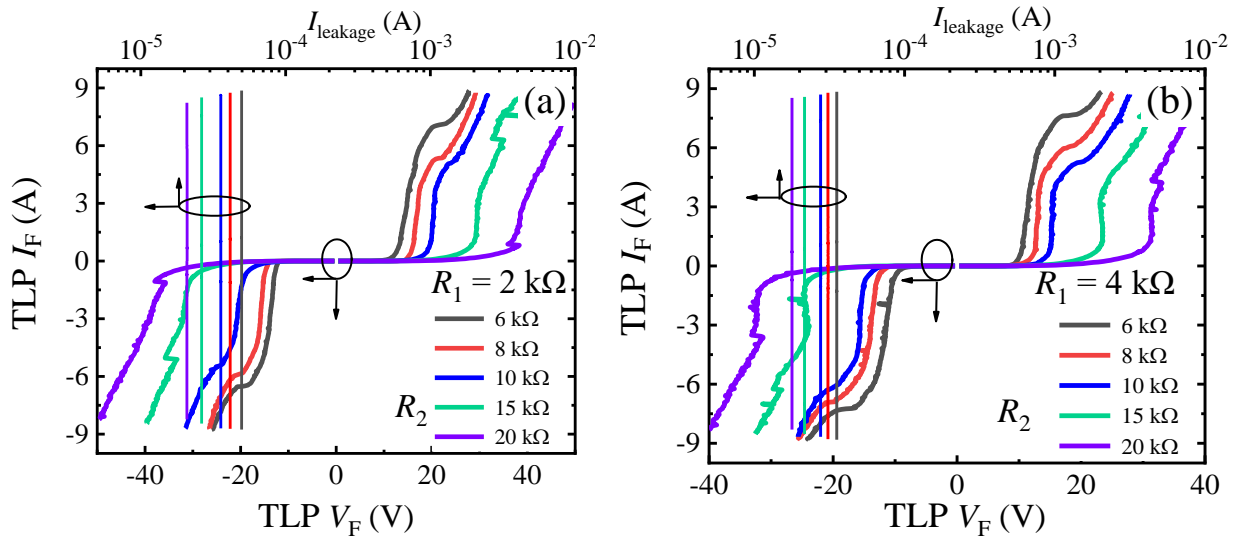

Figure 7. The bidirectional TLP $I-V$ characteristics of the proposed B-TVS-D with different $R_{2}$ : (a) $R_{1 \mathrm{C}} / R_{1 \mathrm{~A}}=2 \mathrm{k} \Omega$; (b) $R_{1 \mathrm{C}} / R_{1 \mathrm{~A}}=4 \mathrm{k} \Omega$.
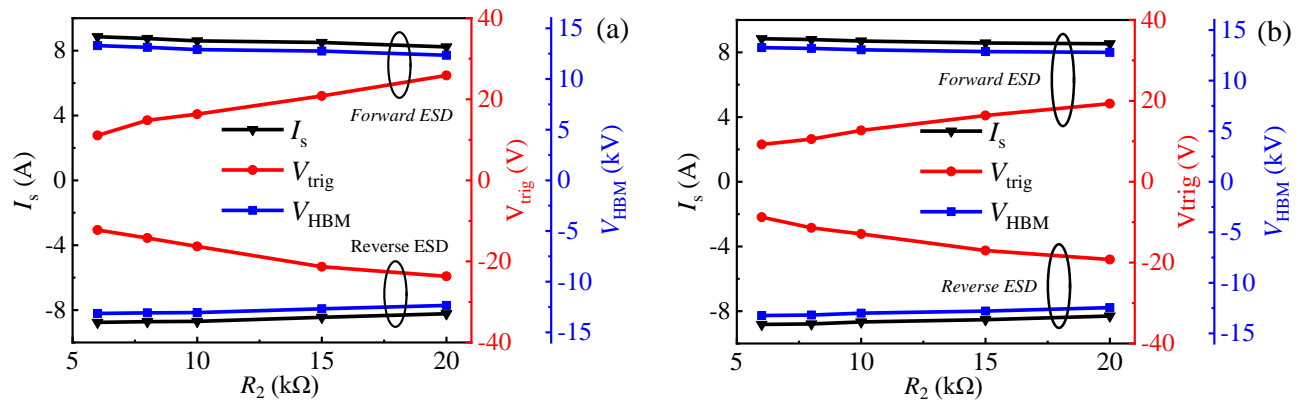

Figure 8. $I_{\mathrm{S}}, V_{\text {trig }}$ and $V_{\mathrm{HBM}}$ of the proposed B-TVS-D with different $R_{2}$ : (a) $R_{1 \mathrm{C}} / R_{1 \mathrm{~A}}=2 \mathrm{k} \Omega$; (b) $R_{1 \mathrm{C}} / R_{1 \mathrm{~A}}=4 \mathrm{k} \Omega$. 
The influence of $R_{1 \mathrm{C}} / R_{1 \mathrm{~A}}$ on the bidirectional static leakage and TLP I-V characteristics of the proposed B-TVS-D is presented in Figures 9 and 10. The diode's $V_{\mathrm{T}}$ is decreased with the increase in $R_{1 \mathrm{C}} / R_{1 \mathrm{~A}}$. With $R_{1 \mathrm{C}} / R_{1 \mathrm{~A}}$ increased from $1 \mathrm{k} \Omega$ to $5 \mathrm{k} \Omega, V_{\mathrm{T}}$ is decreased from $18.2 \mathrm{~V}$ to $6.9 \mathrm{~V}$ for the proposed B-TVS-D with $R_{2}=10 \mathrm{k} \Omega$. This is because the increase in $R_{1}$ will increase the voltage drop at $R_{1 \mathrm{C}}$ and $R_{1 \mathrm{~A}}$, subsequently increasing the voltage at the second gate structure in the forward conduction state or increasing the voltage at the first gate structure in the reverse conduction state. Therefore, a lower applied voltage is needed to turn on the normally-off gate structure of the AlGaN/GaN MBS in the proposed B-TVS-D. Through changing $R_{1}$, a desirable $V_{\mathrm{T}}$ can also be acquired for the proposed B-TVS-D. Similarly, in the ESD event, the triggering voltages of the proposed B-TVS-D are also decreased with the increase in $R_{1}$. With $R_{1 \mathrm{C}} / R_{1 \mathrm{~A}}$ increased from $1 \mathrm{k} \Omega$ to $5 \mathrm{k} \Omega$, the triggering voltages are increased from $23.2 \mathrm{~V}$ to $10.8 \mathrm{~V}$ for the proposed B-TVS-D with $R_{2}=10 \mathrm{k} \Omega$. Through changing $R_{1}$, a desirable triggering voltage can also be acquired for the proposed B-TVS-D (Figure 10). It should be noted that although increasing $R_{1 \mathrm{C}} / R_{1 \mathrm{~A}}$ will lead to a low triggering voltage, it will also reduce response speed of the proposed B-TVS-D, as stated in our previous work [18].
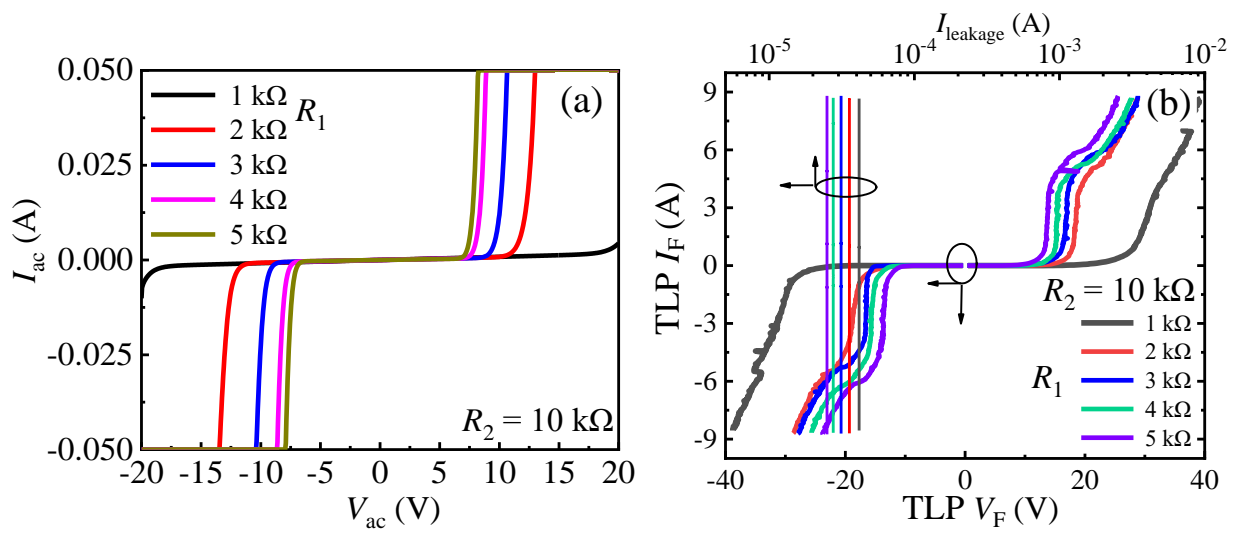

Figure 9. The bidirectional static leakage (a) and TLP I- $V$ characteristics (b) of the proposed B-TVS-D with different $R_{1 \mathrm{C}} / R_{1 \mathrm{~A}} \cdot R_{2}=10 \mathrm{k} \Omega$.

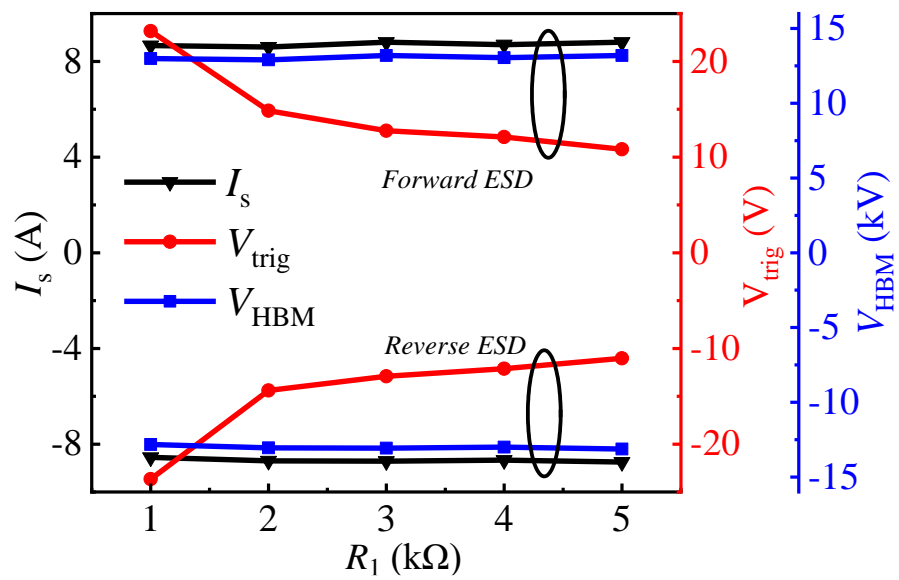

Figure 10. $I_{\mathrm{S}}, V_{\text {trig }}$ and $V_{\mathrm{HBM}}$ of the proposed B-TVS-D with different $R_{1 \mathrm{C}} / R_{1 \mathrm{~A}} \cdot R_{2}=10 \mathrm{k} \Omega$.

\section{Conclusions}

In summary, a bidirectional AlGaN/GaN TVS diode is proposed and investigated. The proposed B-TVS-D features a small-size AlGaN/GaN monolithic bidirectional switch, two current-limiting resistors in parallel connection between the gate electrodes and the neighboring ohmic-contact electrodes (anode/cathode), and a proportional amplification resistor in parallel connection between two gate electrodes. It is demonstrated that the proposed B-TVS-D can be triggered by a desirable voltage and possesses an IS over $8 \mathrm{~A}$ 
(corresponding to $12 \mathrm{kV} V_{\mathrm{HBM}}$ ) in different directional transient electrostatic discharge events. Further, it is also verified that $R_{1 \mathrm{~A}} / R_{1 \mathrm{C}}$ and $R_{2}$ play an important role in the triggering voltage of the proposed B-TVS-D. An increase in $R_{1 \mathrm{~A}} / R_{1 \mathrm{C}}$ or decrease in $R_{2}$ can lead to decrease of the triggering voltage. In addition, the proposed B-TVS-D can be fabricated on the traditional p-GaN HEMT platform, making the ESD design of the GaN-based power system more convenient.

Author Contributions: Conceptualization, Y.S. and Z.H.; methodology, Y.S., Z.H. and H.W.; validation, Y.S., Z.H., L.W. and Y.X.; formal analysis, Y.S. and Z.H.; investigation, Y.S. and L.W.; resources, Y.S. and Y.C.; data curation, Y.S., Y.X., Z.H. and Y.C.; writing-original draft preparation, Y.S. and Z.H.; writing-review and editing, Y.S. and Z.H.; visualization, Y.S.; supervision, G.L. and Y.H.; project administration, Y.S.; funding acquisition, Y.S. All authors have read and agreed to the published version of the manuscript.

Funding: This research was supported by the National Natural Science Foundation of China (NSFC), grant number 62004046, the Key-Area Research and Development Program of Guangdong Province under Grant 2020B010173001, and the Basic scientific research projects in Guangzhou, grant number 202102020317.

Institutional Review Board Statement: Not applicable.

Informed Consent Statement: Not applicable.

Data Availability Statement: Data available on request, having regard to restrictions, e.g., privacy or ethical.

Conflicts of Interest: The authors declare no conflict of interest.

\section{References}

1. Chen, K.J.; Häberlen, O.; Lidow, A.; Tsai, C.L.; Ueda, T.; Uemoto, Y.; Wu, Y. GaN-on-Si Power Technology: Devices and Applications. IEEE Trans. Electron Devices 2017, 64, 779-795. [CrossRef]

2. Shi, Y.; Huang, S.; Bao, Q.; Wang, X.; Wei, K.; Jiang, H.; Li, J.; Zhao, C.; Li, S.; Zhou, Y.; et al. Normally OFF GaN-on-Si MIS-HEMTs Fabricated with LPCVD-SiNx Passivation and High-Temperature Gate Recess. IEEE Trans. Electron Devices 2016, 63, 614-619. [CrossRef]

3. Qin, Y.; Chai, C.; Li, F.; Liang, Q.; Wu, H.; Yang, Y. Study of Self-Heating and High-Power Microwave Effects for EnhancementMode p-Gate GaN HEMT. Micromachines 2022, 13, 106. [CrossRef] [PubMed]

4. Chakraborty, S.; Kim, T.-W. Comprehensive Schottky Barrier Height Behavior and Reliability Instability with Ni/Au and $\mathrm{Pt} / \mathrm{Ti} / \mathrm{Pt} / \mathrm{Au}$ on AlGaN/GaN High-Electron-Mobility Transistors. Micromachines 2022, 13, 84. [CrossRef] [PubMed]

5. Shrestha, N.M.; Li, Y.; Suemitsu, T.; Samukawa, S. Electrical Characteristic of AlGaN/GaN High-Electron-Mobility Transistors with Recess Gate Structure. IEEE Trans. Electron Devices 2019, 66, 1694-1698. [CrossRef]

6. Tang, Z.; Huang, S.; Tang, X.; Li, B.; Chen, K.J. Influence of AlN Passivation on Dynamic ON-Resistance and Electric Field Distribution in High-Voltage AlGaN/GaN-on-Si HEMTs. IEEE Trans. Electron Devices 2014, 61, 2785-2792. [CrossRef]

7. Shankar, B.; Raghavan, S.; Shrivastava, M. Distinct Failure Modes of AlGaN/GaN HEMTs Under ESD Conditions. IEEE Trans. Electron Devices 2020, 67, 1567-1574. [CrossRef]

8. Shankar, B.; Rahgavan, S.; Shrivastava, M. ESD Reliability of AlGaN/GaN HEMT Technology. IEEE Trans. Electron Devices 2019, 66, 3756-3763. [CrossRef]

9. Rossetto, I.; Meneghini, M.; Barbato, M.; Rampazzo, F.; Marcon, D.; Meneghesso, G.; Zanoni, E. Demonstration of field-and power-dependent ESD failure in AlGaN/GaN RF HEMTs. IEEE Trans. Electron Devices 2015, 62, 2830-2836. [CrossRef]

10. Ren, Y.; Chen, Y.Q.; Liu, C.; Xu, X.B.; Gao, R.; Lei, D.Y.; Lai, P.; Huang, Y.; He, J. Gate Failure Behavior and Mechanism of AlGaN/GaN HEMTs Under Transmission Line Pulsed Stress. IEEE J. Electron Devices Soc. 2021, 9, 628-632. [CrossRef]

11. Chen, S.H.; Griffoni, A.; Srivastava, P.; Linten, D.; Thijs, S.; Scholz, M.; Denis, M.; Gallerano, A.; Lafonteese, D.; Concannon, A HBM ESD robustness of GaN-on-Si Schottky diodes. IEEE Trans. Device Mater. Reliab. 2012, 12, 589-598. [CrossRef]

12. Shankar, B.; Sengupta, R.; Gupta, S.D.; Soni, A.; Shrivastava, M. On the ESD Behavior of AlGaN/GaN Schottky Diodes and Trap Assisted Failure Mechanism. In Proceedings of the 39th Electrical Overstress/Electrostatic Discharge Symposium (EOS/ESD) 2017, Tucson, AZ, USA, 10-14 September 2017.

13. Wang, Z.; Liou, J.; Cho, K.; Chiu, H. Development of an Electrostatic Discharge Protection Solution in GaN Technology. IEEE Electron Device Lett. 2013, 34, 1491-1493. [CrossRef]

14. Shankar, B.; Singh, R.; Sengupta, R.; Khand, H.; Soni, A.; Gupta, S.; Raghavan, S.; Gossner, H.; Shrivastava, M. Trap Assisted Stress Induced ESD Reliability of GaN Schottky Diodes. In Proceedings of the 40th Electrical Overstress/Electrostatic Discharge Symposium (EOS/ESD) 2018, Reno, NV, USA, 23-28 September 2018.

15. Chang, S.J.; Chen, C.H.; Su, Y.K.; Sheu, J.K.; Lai, W.C.; Tsai, J.M.; Liu, C.H.; Chen, S.C. Improved ESD protection by combining InGaN-GaN MQW LEDs with GaN Schottky diodes. IEEE Electron Device Lett. 2003, 24, 129-131. [CrossRef] 
16. Xin, Y.; Chen, W.; Sun, R.; Shi, Y.; Liu, C.; Xia, Y.; Wang, F.; Xu, X.; Shi, Q.; Wang, Y.; et al. Electrostatic Discharge (ESD) Behavior of p-GaN HEMTs. In Proceedings of the 32nd International Symposium on Power Semiconductor Devices and ICs (ISPSD), Vienna, Austria, 13-18 September 2020.

17. Ker, M.-D.; Peng, J.-J.; Jiang, H.-C. ESD test methods on integrated circuits: An overview. In Proceedings of the IEEE International Conference on Electronics, Circuits and Systems (ICECS), Malta, Malta, 2-5 September 2001.

18. Xin, Y.; Chen, W.; Sun, R.; Wang, F.; Deng, X.; Li, Z.; Zhang, B. Simulation Study of a High Gate-to-Source ESD Robustness Power p-GaN HEMT with Self-Triggered Discharging Channel. IEEE Trans. Electron Devices 2021, 68, 4536-4542. [CrossRef]

19. Yao, B.; Shi, Y.; Wang, H.; Xu, X.; Chen, Y.; He, Z.; Xiao, Q.; Wang, L.; Lu, G.; Li, H.; et al. A Novel Bidirectional AlGaN/GaN ESD Protection Diode. Micromachines 2022, 13, 135. [CrossRef]

20. Gansystems. Available online: https://gansystems.com/gan-transistors/ (accessed on 1 September 2015).

21. EPC. eGaN FET Datasheet. Available online: https://epcco.com/epc/Portals/0/epc/documents/datasheets/EPC2036_ datasheet.pdf (accessed on 1 January 2021). 